\title{
РОЛЬ АНТИТЕЛ К РЕЦЕПТОРУ ТИРЕОТРОПНОГО ГОРМОНА В ФОРМИРОВАНИИ ФУНКЦИОНАЛЬНЫХ НАРУШЕНИЙ ЩИТОВИДНОЙ ЖЕЛЕЗЫ У НОВОРОЖДЕННЫХ ОТ МАТЕРЕЙ С ДИФФУЗНЫМ ТОКСИЧЕСКИМ ЗОБОМ
}

\author{
'Ворохобина Н.В., ' Кузнецова А.В., ${ }^{2}$ Ловкова Ю.С. \\ 'ФГБОУ ВО «Северо-Западный государственный медицинский университет имени И.И. Мечникова» Минздрава России, \\ Санкт-Петербург \\ ${ }^{2}$ СПбГБУз «Родильный дом № 6 имени профессора В.Ф. Снегирева, Санкт-Петербург
}

ЦЕЛЬ: изучить функциональное состояние щитовидной железы (ЩЖ) у новорожденных от матерей с диффузным токсическим зобом (ДТЗ), в зависимости от уровня антител к рецепторам тиреотропного гормона (АТ-рТТГ).

МАТЕРИАЛЫ И МЕТОДЫ: обследовано 68 новорожденных от 67 матерей с ДТЗ. В группУ контроля вошли 49 новорожденных от 49 матерей без патологии ЩЖ. Для оценки функционального состояния ЩЖ новорожденных определяли уровни тиреотропного гормона (ТТГ), свободного тироксина (свТ 4 ), АТ-рТТГ в пуповинной крови и высчитывали коэффициент ТТГ/свТ ${ }_{4}$. На 4-7-й день жизни всем новорожденным проводилось ультразвуковое исследование (УЗИ) ЩЖ.

PЕЗУЛЬТАТЫ: повышенное содержание АТ-рТТГ в сыворотке крови беременных женщин с ДТЗ и в пуповинной крови новорожденных ассоциировалось с гиперплазией ЩЖ, более низкими росто-весовыми показателями и развитием неонатального тиреотоксикоза.

ВЫВоды: полученные результаты свидетельствуют о необходимости тщательной оценки режимов антитиреоидной терапии у беременных с ДТЗ, а также мониторинга уровня АТ-рТТГ в сыворотке крови данных пациенток во время беременности с целью снижения риска нарушения функции ЩЖ у плода и новорожденного. Контроль уровня АТ-рТТГ должен проводиться на этапе планирования беременности и при ее наступлении у пациенток с ДТЗ 1 раз в триместр при нормальных значениях, а в случае повышения - 1 раз в месяц.

В исследовании показано, что новорожденные от матерей с ДТЗ имели более низкие росто-весовые показатели по сравнению с новорожденными от матерей контрольной группы. Средний уровень АТ-рТТГ в пуповинной крови у новорожденных от матерей с ДТ3 превышал нормальные значения новорожденных от матерей без патологии ЩЖ. Максимальные значения АТ-рТТГ отмечены у детей, рожденных от матерей с рецидивом тиреотоксикоза во время беременности. Подтверждена прямая корреляционная связь между уровнем АТ-рТТГ в крови беременных, особенно во II и III триместрах, и их содержанием в пуповинной крови новорожденных. У новорожденных от матерей с рецидивирующим течением ДТЗ и повышенным уровнем АТ-рТТГ в пуповинной крови размеры ЩЖ были больше, чем у новорожденных из контрольной группы. Максимальные размеры ЩЖ выявлены у детей от матерей, получавших тиреостатики в течение всей беременности. Выявленная у 16,2\% новорожденных субклиническая гиперфункция ЩЖ связана с трансплацентарным переходом АТ-рТТГ, что подтверждено значительным их преобладанием у этих новорожденных. Обнаружена прямая положительная связь между уровнем АТ-рТТГ в пуповинной крови и частотой возникновения неонатального тиреотоксикоза.

КЛЮчЕВЫЕ СЛОВА: диффузный токсический зоб, щитовидная железа, антитела к речептору тиреотропного гормона, беременность, новорожденные, неонатальный тиреотоксикоз. 